\title{
Jetting behavior in drop-on-demand printing: Laboratory experiments and numerical simulations
}

\author{
E. Antonopoulou ${ }^{\circ}$ \\ EPSRC Centre for Doctoral Training in Fluid Dynamics, University of Leeds, Leeds LS2 9JT, United Kingdom \\ O. G. Harlen \\ Department of Applied Mathematics, University of Leeds, Leeds LS2 9JT, United Kingdom \\ M. A. Walkley (0) \\ School of Computing, University of Leeds, Leeds LS2 9JT, United Kingdom \\ N. Kapur $\odot$ \\ School of Mechanical Engineering, University of Leeds, Leeds LS2 9JT, United Kingdom
}

(Received 22 November 2019; accepted 18 March 2020; published 29 April 2020)

\begin{abstract}
The formation and evolution of micron-sized droplets of a Newtonian liquid generated on demand in an industrial inkjet printhead are studied experimentally and simulated numerically. The shapes and positions of droplets during droplet formation are observed using a high-speed camera and compared with their numerically obtained analogs. Both the experiments and the simulations use practical length scales for inkjet printing. The results show how fluid properties, specifically viscosity and surface tension, affect the drop formation, ligament length, and breakoff time. We identify the parameter space of fluid properties for producing single drops at a prescribed speed and show this is not simply a restriction on the Ohnesorge number, but that there is an additional restriction on the Reynolds number that is distinct from the Reynolds number limit associated with the prevention of splashing. This phase diagram provides more precise guidance on the space of fluid parameters for jetting single droplets in drop-on-demand inkjet printers.
\end{abstract}

DOI: 10.1103/PhysRevFluids.5.043603

\section{INTRODUCTION}

Industrial inkjet printing requires precise control of the formation and jetting of small droplets of liquid ink. A typical industrial drop-on-demand (DOD) printhead contains hundreds to thousands of nozzles arranged in an array such that each nozzle can be independently controlled. Nozzle diameters range from 10 to $100 \mu \mathrm{m}$ in diameter, generating drops with a volume from 0.5 to $500 \mathrm{pL}$ at speeds typically between 5 and $10 \mathrm{~m} / \mathrm{s}$ [1,2]. A key challenge in DOD printing is to identify the combinations of printhead design, actuation signals, and fluid properties to generate droplets of a specified speed and size while minimizing the formation of satellite droplets. In this paper we use a combination of numerical simulations and experiments to identify the parameter space of fluid properties for generating single droplets at the typical speed and scale found in inkjet printing.

In the majority of industrial inkjet printers drops are generated by a piezoelectric ceramic element, which changes shape in response to an electric current, creating a pressure pulse within

*scea@leeds.ac.uk 
the printhead that leads to the ejection of a small volume of fluid [2,3]. The shape that is commonly observed for the ejected fluid upon exiting the nozzle is a nearly spherical head with a trailing ligament $[2,4]$. However, the detailed shape, drop volume, and velocity are all controlled by the magnitude and form of the pressure pulse.

A low-amplitude pressure pulse produces a slow drop with a short ligament that is absorbed into the head forming a single drop. Increasing the amplitude of the pulse results in a higher drop speed, but also a longer ligament. Since such ligaments are unstable to the Rayleigh-Plateau [5,6] instability, longer ligaments (above a critical aspect ratio) break up into smaller satellite drops [7]. While some, faster moving, satellite drops will catch up and merge with the main drop, satellite drops that do not merge tend not to land at the desired location and so are detrimental to print quality. Therefore, although increasing the speed of the main drop improves both printing accuracy and productivity, it can also lead to an increase in satellite drop generation. For Newtonian fluids, the formation of droplets from jets can be characterized by two dimensionless groups comparing the importance of surface tension, viscosity, and inertia. These are the Reynolds number Re, the ratio between inertial and viscous forces in a moving fluid,

$$
\operatorname{Re}=\frac{\rho U R}{\mu},
$$

where $\rho$ is the ink density, $U$ is the drop speed, $R$ is the nozzle radius, and $\mu$ is the ink viscosity; and the Weber number We, that is, the ratio between inertia and surface tension

$$
\mathrm{We}=\frac{\rho U^{2} R}{\gamma},
$$

where $\gamma$ is the ink surface tension. However, these two dimensionless groups can be combined to form a further group, the Ohnesorge number Oh [8], which is independent of drop speed and depends only on the physical properties of the liquid and the dimensions of the jet or the drop,

$$
\mathrm{Oh}=\frac{\sqrt{\mathrm{We}}}{\operatorname{Re}}=\frac{\mu}{\sqrt{\gamma \rho R}} .
$$

The Ohnesorge number characterizes both the capillary driven pinch-off of a liquid bridge, which causes the breakoff of the jet from the nozzle, and the Rayleigh-Plateau instability of an infinite jet, which is the key mechanism for satellite drop formation. In particular, Derby [9] suggests that DOD printing requires the value of Ohnesorge number to be in the range $0.1-1$. For $\mathrm{Oh}>1$ viscosity delays the breakoff of the jet from the nozzle, while for $\mathrm{Oh}<0.1$ the ligament will break up to form a large number of satellite droplets. While this criterion is a useful guide in the development of inks, it does not take account of the effects of drop speed on ligament length, which in turn affects the formation of satellites [7].

The inks used in the inkjet printing industry are often complex formulations including particulates, polymers, and other additives depending on the functional requirements of the ink $[9,10]$. These additives can cause the inks to have complex rheological behavior under the high-strain-rate conditions of inkjet printing. For example polymeric additives have been shown to have a dramatic effect $[11,12]$ on drop formation, due to the increased resistance to extensional flow. The presence of particulates increases the fluid viscosity, but can also introduce other effects, for example, where an asymmetry of the meniscus leads to the entrapment of air bubbles inside the nozzle [13]. However, in this paper we focus on the jetting of Newtonian fluids that have constant surface tension and viscosity.

Detailed studies of the contraction of cylindrical fluid filaments [14-17] find that the Ohnesorge number indicates the critical aspect ratio that determines whether a filament contracts to a single drop or breaks up due to droplets breaking away from the end of the filament. For Ohnesorge numbers greater than around 0.3 , a fluid filament of aspect ratio 25 contracts to a single drop, whereas for smaller Ohnesorge numbers the filament will break into multiple droplets [15-17]. 


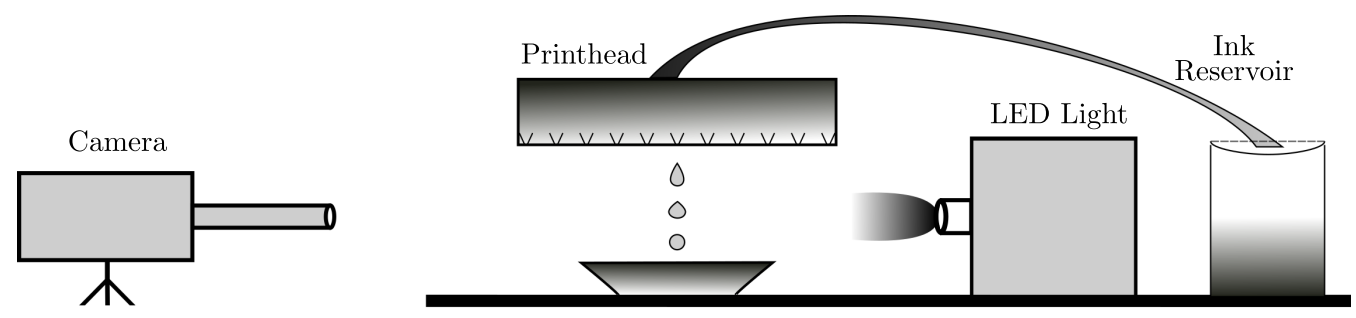

FIG. 1. Schematic diagram of the experimental setup.

There have been a number of experimental studies of DOD jetting [3,18-22,29] in which the pressure and velocity response to the electric signal inside the printhead have been measured in response to the electrical driving pulse along with the meniscus position and minimum jet radius. Castrejón-Pita et al. [23] compared simulations with a large-scale experimental model of a drop-on-demand printer and found excellent agreement in the detailed predictions of the evolution of the fluid domain during drop formation. In their experiments the nozzle diameter was $2 \mathrm{~mm}$; however, the fluid properties at jetting speeds were chosen such that the Reynolds and Weber numbers were representative of industrial inkjet printing. Experimental images of droplet formation were compared with numerical simulations using the Lagrangian finite-element code of Morrison and Harlen [11] and showed excellent agreement between the simulations and experiments.

In this paper we extend this earlier study [23] and the more recent work of Wang et al. [24] in two ways. We explore in further detail the parameter space in which the desired jetting behavior for inkjet printing is found. In particular, we focus on a range of values for surface tension and viscosity required to produce drops at a desired drop speed from a given nozzle. We also compare our simulations with high-speed video observations of drop formation in an industrial inkjet printhead rather than a large-scale model to determine whether the different driving mechanics and more complex structure of a commercial printhead affect the comparison. These experiments are complementary to those of Dong et al. $[18,30]$ as we explore more viscous fluids and the use of high-speed video allows us to look at the variability between drops. We then examine the dependence of different jetting properties, such as ligament length and breakoff time, on fluid parameters and jetting speed. Finally, we present a phase diagram which predicts a region for good jetting behavior parametrized by viscosity and surface tension. Our study shows that to achieve a satellite-free drop, there is an additional requirement that the Reynolds number should be below 10, in addition to the restriction on the Ohnesorge number suggested by previous studies.

\section{METHODS}

\section{A. Experimental details}

The experiments were performed using a Ricoh GEN-5 printhead (RICOH) which uses a piezoelectric drive to generate a pressure pulse in the ink chamber causing liquid to be ejected from the nozzle orifice (Fig. 1). In this work the waveform of the voltage (and therefore pressure) signal was kept constant, with amplitude adjusted to control the speed of ejected fluid. Other studies have investigated the effect of the waveform optimization in the droplet formation showing key characteristics of the timing and the amplitude in different jetting properties [18]. The simulation code used in this work requires an equivalent velocity waveform. This was inferred from the flow rate at the nozzle orifice. More details of the waveform used in the simulations are discussed in Sec. II B 1.

High-speed videos were recorded using an ultrahigh-speed Photron Fastcam SA-Z camera. This provided images at a resolution of $512 \times 56$ recorded at 480000 frames per second. This allows the evolution of a single drop to be followed, enabling variations between different jetting events to be 
TABLE I. Properties of the PPH-TPM mixture.

\begin{tabular}{lcc}
\hline \hline PPH & Viscosity (mPa s) & Surface tension $(\mathrm{mN} / \mathrm{m})$ \\
\hline 20 & 7.4 & 35 \\
40 & 9.4 & 36 \\
50 & 11.6 & 37 \\
60 & 12.9 & 38 \\
test fluid & 12 & 30 \\
\hline \hline
\end{tabular}

captured, such as the position of satellites drops, while producing images of sufficient quality for the details of the ligament breakup to be observed.

Experiments were conducted using two different sets of fluids. A test fluid provided by RICOH with a surface tension of $30 \mathrm{mN} / \mathrm{m}$, viscosity $12 \mathrm{mPa} \mathrm{s}$, and density $1021 \mathrm{~kg} \mathrm{~m}^{-3}$ and mixtures of propylene glycol phenyl ether (PPH) and tripropylene glycol methyl ether (TPM) at 20, 40, 50, and 60 wt./vol \% to give a range of fluids with different viscosities but similar surface tensions. The fluid viscosity and surface tension were measured using a Malvern Kinexus rotational rheometer and surface tension on a Biolin Scientific Theta Attension tensiometer, respectively, with results presented in Table I. As expected, all the fluids showed a Newtonian behavior at the measured shear rates. All the experiments were performed at $26^{\circ} \mathrm{C}$.

\section{B. Simulation details}

\section{Governing equations and boundary conditions}

As the temperature of the fluid in the printhead is maintained at a constant value, there are no significant temperature variations during the jetting process, and hence the viscosity and surface tension can be assumed to be constant. We can also neglect the effects of gravity due to the small scales involved [11]. Hence, for a Newtonian fluid the dynamics are described by the Navier-Stokes equation

$$
\rho \frac{D \mathbf{u}}{D t}=-\nabla p+\mu \nabla \cdot\left[\nabla \mathbf{u}+(\nabla \mathbf{u})^{T}\right],
$$

where $\mathbf{u}$ is the fluid velocity, $p$ is the pressure in the nozzle, and $\mu$ is the dynamic viscosity of the fluid together with the condition of incompressibility

$$
\nabla \cdot \mathbf{u}=0
$$

We will not model the detailed flow within the entire printhead, but instead consider only the flow in the region close to the nozzle. Since the nozzle is axisymmetric this allows us to make the assumption of axisymmetry where the axis of symmetry lies at the center of the outlet nozzle, even though the printhead itself is nonaxisymmetric. The shape of the nozzle was chosen to replicate the dimensions of the experimental nozzle. The initial finite-element grid is shown in Fig. 2. The curved inlet (left side of Fig. 2) is an artificial inflow boundary across which there is a mass flow driven by the pressure variations within the printhead.

A time-dependent velocity boundary condition is imposed on this inlet boundary to provide a mass flow with a magnitude given by a driving signal. This is a boundary condition based on the qualitative behavior of the printhead drive, which produces a "pull-push-pull" waveform. A graph of the time dependence of the signal used in the simulations and the corresponding position of the meniscus is shown in Fig. 3. This is formed by three parabolic segments, each of $4 \mu$ s duration. In the initial phase (the first parabolic section) the meniscus is drawn back into the printhead. In the second phase the pressure drives liquid from the reservoir through the nozzle orifice. In the final stage liquid is again drawn back into the nozzle from the tail of the emergent jet. 


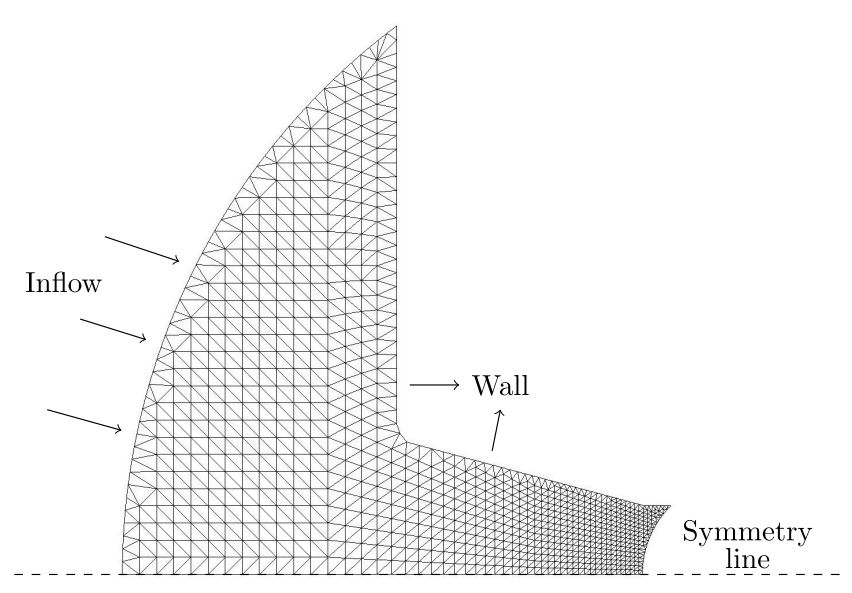

FIG. 2. Nozzle shape and initial mesh used in the simulations. The jet is assumed to be axisymmetric, so for the production of subsequent images, the results are mirrored around the axis of symmetry.

We assume that the contact line is pinned at the nozzle outlet and that no slip occurs at the nozzle walls. Conditions of zero velocity are imposed $(\mathbf{u}=0)$. At the free surface we assume that the drag on a droplet due to air resistance is negligible [20] and impose a boundary condition on the stress $\boldsymbol{\sigma}=-p \mathbf{I}+\mu\left[\nabla \mathbf{u}+(\nabla \mathbf{u})^{T}\right]$ due to surface curvature,

$$
[\boldsymbol{\sigma} \cdot \mathbf{n}]_{\mathrm{air}}^{\mathrm{jet}}=-\gamma\left(\nabla_{s} \cdot \mathbf{n}\right) \mathbf{n}
$$

Here $\gamma$ is the coefficient of surface tension, $\mathbf{n}$ is the unit vector normal to the free surface (directed outward from the jet), and the surface divergence operator is given by $\nabla_{s^{*}}:=\nabla \cdot(\mathbf{I}-\mathbf{n n})$.

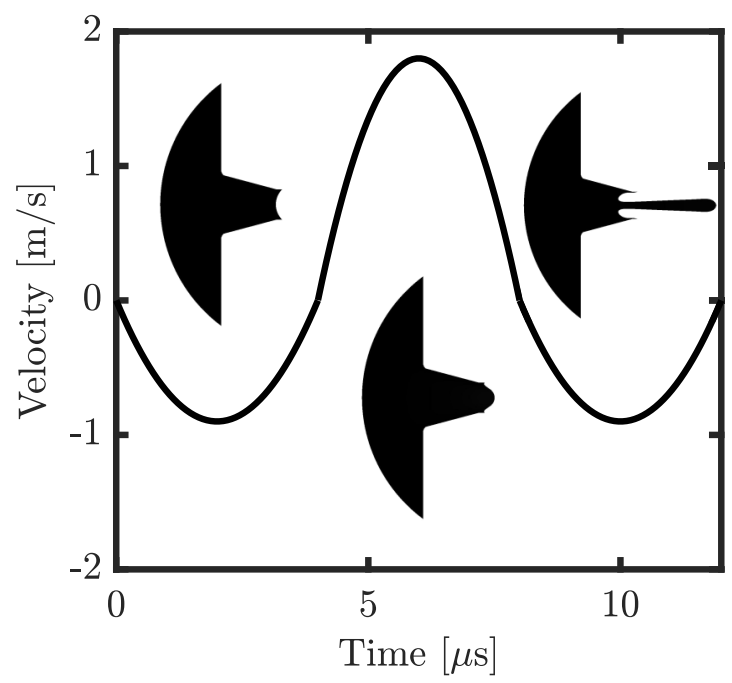

FIG. 3. Plot of the driving signal as a function of time, which is imposed as a flux boundary condition over the nozzle inlet. Images show the meniscus position at 0,6 , and $11 \mu \mathrm{s}$. 
The equations can be put into dimensionless form by scaling lengths with the nozzle outlet radius $R$, velocities by the drop speed $U$, and pressure and stress by $\rho U^{2}$. These scalings yield the dimensionless governing equations for the fluid domain $\Omega$,

$$
\begin{gathered}
\frac{D \mathbf{u}}{D t}-\nabla \cdot \sigma=0, \\
\nabla \cdot \mathbf{u}=0,
\end{gathered}
$$

where $t, \mathbf{u}$, and $\sigma$ are now the dimensionless time, velocity, and stress, respectively, with the stress tensor now given by

$$
\boldsymbol{\sigma}=-p \mathbf{I}+\frac{1}{\operatorname{Re}}\left[\nabla \mathbf{u}+(\nabla \mathbf{u})^{T}\right]
$$

The dimensionless interface boundary condition on the free surface $\Gamma$ is given by

$$
[\boldsymbol{\sigma} \cdot \mathbf{n}]_{\text {air }}^{\mathrm{jet}}=-\frac{1}{\mathrm{We}}\left(\nabla_{s} \cdot \mathbf{n}\right) \mathbf{n} .
$$

\section{Numerical method}

The governing equations (3) and (4) together with boundary condition (6) were solved using a moving grid finite-element method, which was first developed for the study of creeping flow of dilute polymer solutions [25] and extended to model DOD printing of both Newtonian [23] and viscoelastic inks [11]. In this method, the finite-element mesh is Lagrangian, which means that the nodes advect with the fluid flow, so the mesh naturally follows the evolution of the free surface. More information on this method can be found in Ref. [25].

a. Weakformulation. We define $\phi_{i}$ and $\psi_{j}$ as the respective velocity and pressure basis functions giving the Galerkin weak formulation, where we have used integration by parts to remove the divergence of stress,

$$
\begin{gathered}
\int_{\Omega} \phi_{i} \frac{D \mathbf{u}}{D t} d \Omega+\int_{\Omega} \nabla \phi_{i} \cdot \sigma d \Omega=\int_{\Gamma} \phi_{i} \boldsymbol{\sigma} \cdot \mathbf{n} d \Gamma, \quad i=1, \ldots, N_{v} \\
\int_{\Omega} \psi_{j}(\nabla \cdot \mathbf{u}) d \Omega=0, \quad j=1, \ldots, N_{p},
\end{gathered}
$$

where $\Gamma$ is the boundary of the domain $\Omega$ and $N_{v}$ and $N_{p}$ are the number of non-Dirichlet velocity and pressure nodes, respectively.

Applying the free-surface boundary condition to the surface integral in Eq. (7) gives

$$
\int_{\Gamma}\left(\phi_{i} \boldsymbol{\sigma} \cdot \mathbf{n}\right) d \Gamma=\int_{\Gamma_{f}} \phi_{i} \frac{1}{\mathrm{We}}\left(\nabla_{s} \cdot \mathbf{n}\right) \mathbf{n} d \Gamma,
$$

as the velocity satisfies a Dirichlet condition on the remaining portion of the boundary. This surface boundary contribution is treated using the method presented by Westborg and Hassager [26]. For an axisymmetric surface, the curvature $-\nabla_{s} \cdot \mathbf{n}$ can be expressed as

$$
-\nabla_{s} \cdot \mathbf{n}=\kappa_{s}+\kappa_{\phi}
$$

where $\kappa_{s}$ is the curvature along the interface and $\kappa_{\phi}$ is the azimuthal curvature. The curvature $\kappa_{s}$ can be evaluated using the Serret-Frenet formula as

$$
\frac{d \mathbf{t}}{d s}=-\kappa_{s} \mathbf{n},
$$

where $s$ denotes the arc length along the curve and $\mathbf{t}$ the unit tangent vector in the direction of $s$. Hence, performing the azimuthal integral along a section of the free surface, we obtain

$$
\int_{\Gamma_{A B}} \phi_{i} \frac{1}{\mathrm{We}}\left(\nabla_{s} \cdot \mathbf{n}\right) \mathbf{n} d \Gamma=\frac{2 \pi}{\mathrm{We}} \int_{s_{B}}^{s_{A}}\left(\frac{d \mathbf{t}}{d s}-\kappa_{\phi} \mathbf{n}\right) \phi_{i} r d s
$$


where $s_{A}$ and $s_{B}$ are the values of $s$ at the end point of the interface boundary. Integrating by parts the term involving a second-order derivative yields

$$
\int_{\Gamma_{A B}} \phi_{i} \frac{1}{\mathrm{We}}\left(\nabla_{s} \cdot \mathbf{n}\right) \mathbf{n} d \Gamma=\left.\frac{2 \pi}{\mathrm{We}}\left[\mathbf{t} \phi_{i} r\right]\right|_{s_{B}} ^{s_{A}}-\frac{2 \pi}{\mathrm{We}} \int_{s_{B}}^{s_{A}}\left(\mathbf{t} \frac{d}{d s}\left(r \phi_{i}\right)+\mathbf{n} \phi_{i} r \kappa_{\phi}\right) d s .
$$

With this formulation, we can represent the interface with functions requiring only $C^{0}$ continuity. The first term on the right-hand side of Eq. (13) vanishes if the surface contact line is pinned since there is no velocity unknown for which $\phi_{i}$ is nonzero at the contact point. However, this term must be included when considering a dynamic contact line.

$b$. Time discretization. Time derivatives are evaluated using a $\theta$ scheme where the value of a variable $\psi$ at the $(n+1)$ th time step is given by

$$
\psi_{n+1}=\psi_{n}+\delta t\left[\theta \dot{\psi}_{n+1}+(1-\theta) \dot{\psi}_{n}\right],
$$

where $\delta t$ is the time step, $\theta \in[0,1]$ is the weighting parameter of the scheme, and $\dot{\psi}=d \psi / d t$. In addition to the time derivative in the momentum equation, the solution at the $(n+1)$ th step depends upon the position of the nodes, which move with the fluid velocity. Since the node positions depend on the solution for the velocity, a Picard iteration scheme is used to determine the future positions of the nodes.

c. Pinch-off. The event of capillary breakup of the fluid thread connecting the main droplet to the printhead is a point of singularity of the Navier-Stokes equations. Therefore, although simulations with sufficient resolution can approach this point, they cannot capture the change in surface topology when the fluid domain is broken into separate domains. In keeping with previous work [23], we artificially cut the domain at a point where the minimum jet radius is below a certain threshold. Here the threshold is taken to be less than $1 \%$ of the nozzle outlet radius, as this is beyond the point where the thinning follows the universal capillary pinching solution [27]. We have verified that this value is sufficient to capture the dynamics by comparing with simulations using more refined meshes and lower values for the cutoff threshold, where we found no significant changes to the breakoff time, ligament length, and drop speed.

Once broken off, the separate fluid domains do not interact. This means that we do not capture coalescence events that occur when faster moving satellite drops collide with slower moving drops in front of them. This can be seen, for example, in the bottom frame in Fig. 5, where there appears to be a satellite in front of the main drop, which would have merged with the main drop.

\section{RESULTS}

\section{A. Comparison between simulations and experiments}

We begin by comparing the images of drop formation between the simulations and experiments for droplets jetted at $7 \mathrm{~m} / \mathrm{s}$, which is considered the optimal printing speed for this printhead. These are shown in Figs. 4 and 5, where we compare snapshots at different times after the drop emerges from the nozzle. Each image compares two different experimental droplets produced from the same nozzle under the same jetting conditions with the simulation where the ink properties at drop speed match those of the experiments presented in Sec. II A. The principle uncertainty in the comparison is the precise form of the driving waveform.

Nevertheless, it can be seen that there is good agreement between the experiments and the simulations. In particular, the first snapshot, taken shortly after the ligament detaches from the printhead, shows that this first breakoff event is captured accurately by the simulations. Following breakoff the ligament shortens with a bulb forming at the end. In the final frame we see the growth of variations in the filament thickness that lead to breakup of the filament into satellites. Comparing the final frames in Fig. 4, we observe that the precise position of these bulges on the filament differs between the left- and right-hand image, indicating that these arise from the growth of instabilities seeded by noise that varies between droplets. Similar variations in thickness are seen in 


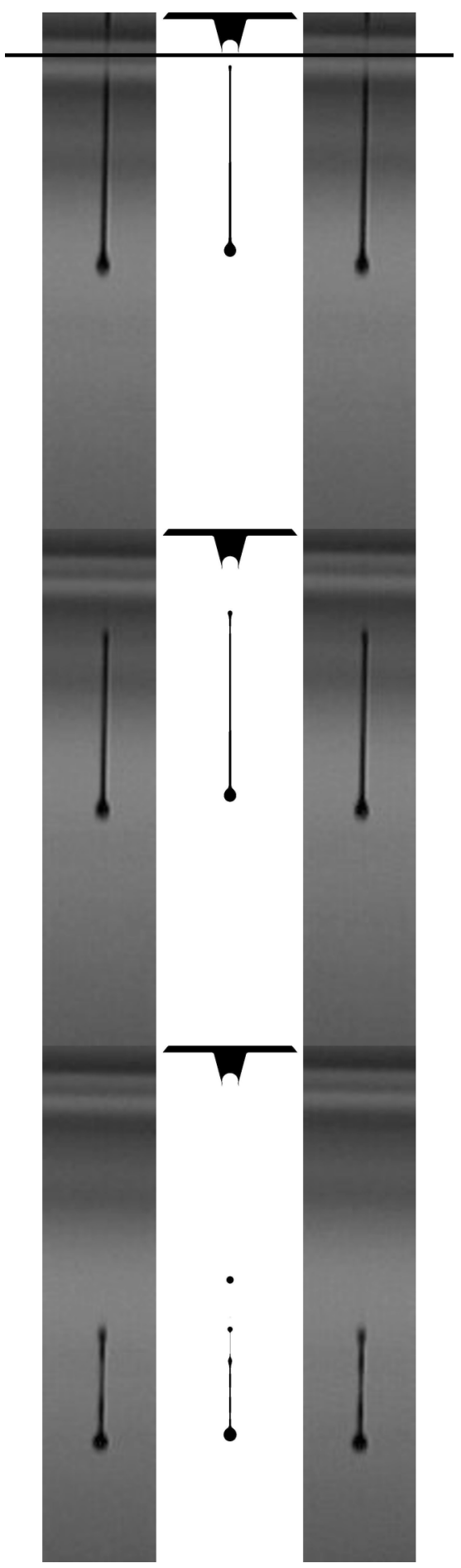

FIG. 4. Comparison between simulations (white background) and experiments (grey background) with test fluid (RICOH) at different times for a prescribed drop speed of $7 \mathrm{~m} / \mathrm{s}$ at 41,48 , and $77 \mu \mathrm{s}$, from top to bottom. The solid black line in the top picture indicates the position of the nozzle plate. Here we present two different experimental droplets produced from the same nozzle under the same jetting conditions. 


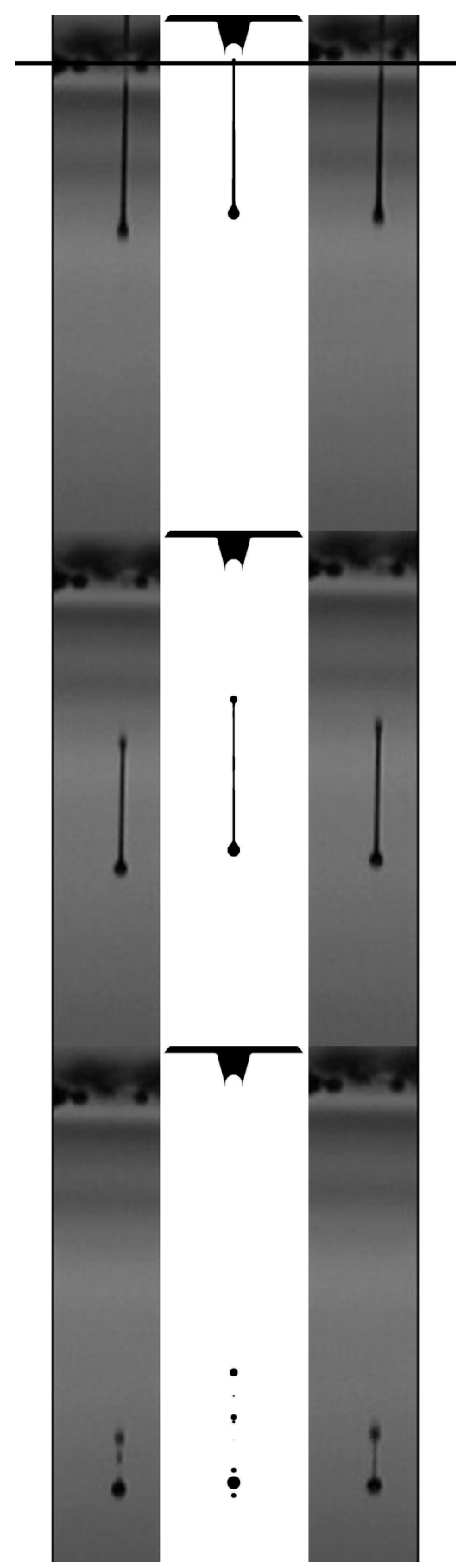

FIG. 5. Comparison between simulations (white background) and experiments (gray background) with a PPH $40 \%$ mixture at different times for a prescribed drop speed of $7 \mathrm{~m} / \mathrm{s}$ at 33,60 , and $89 \mu \mathrm{s}$, from top to bottom. The solid black line indicates the position of the nozzle plate. Note that the appearance of a satellite below the main drop at the longest elapsed time is an artifact of the simulation method and would in practice have coalesced with the main drop. 


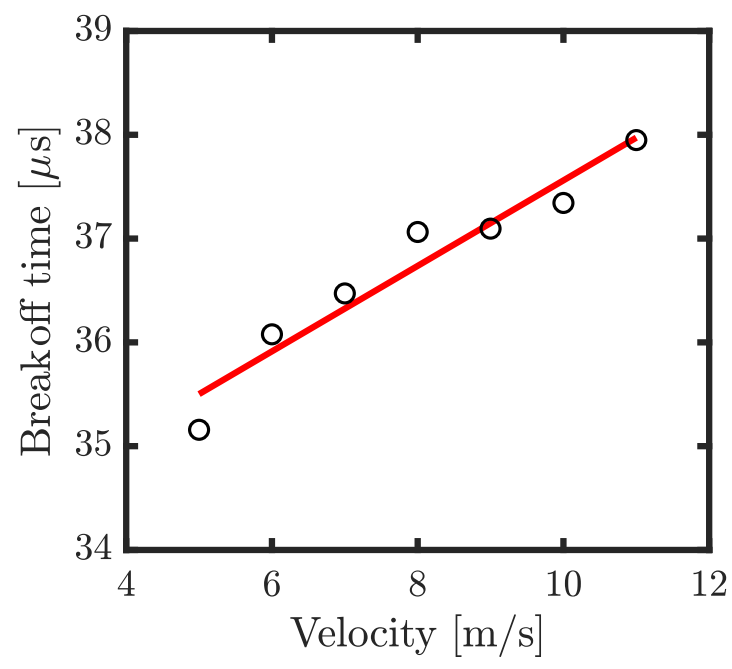

FIG. 6. Graph showing the time of breakoff of the droplet from the nozzle as a function of drop velocity from simulations. Time is measured from the start of the drive signal. The solid line shows a linear fit to the data and shows that the breakoff time has a weak linear increase with the drop velocity.

the simulations, although we observe an earlier breakup of the tail of the filament in the simulations than is seen experimentally.

The 40\% PPH solution, shown in Fig. 5, shows a qualitatively similar evolution. However, in the final frame it can be seen both that the ligament is shorter and that the instability has developed further to the point where the ligament of the left-hand image has broken into two satellite drops. This fluid has both a lower viscosity and a higher surface tension than the RICOH test fluid and so has a lower Ohnesorge number. Again, the ligament in the simulation breaks up slightly earlier than in the experiments. This may be a consequence of the approximation of the drive waveform. Note also that the appearance of a satellite below the main drop at the longest elapsed time is an artifact of the simulation method and would in practice have coalesced with the main drop. Nevertheless, the overall level of agreement confirms that our numerical model can capture the dynamics of jet breakup in a commercial inkjet printhead.

\section{B. Effect of jetting speed}

We next examine the effects of varying the jetting speed, by varying the amplitude of the drive waveform (or in the case of the experiments, the voltage applied to the piezoelectric drive). The ink properties for the simulations were chosen to match the ones of the test fluid, described in Sec. II A. The velocity of the drop increases nearly linearly with the amplitude, in agreement with previous experimental studies [1]. In inkjet printing the desired range of the drop velocity is usually $5-10 \mathrm{~m} / \mathrm{s}$, to prevent drops splashing on impact.

We define the breakoff time as the time interval between the start of the drive waveform and the breakoff of the droplet from the nozzle as a function of drop velocity in the simulations. In Fig. 6 we see that this increases only slightly with increasing droplet speed (by a factor of $10 \%$ between 5 and $11 \mathrm{~m} / \mathrm{s}$ ), suggesting that breakoff is primarily determined by surface-tension-driven thinning rather than the pressure wave. It can also be observed that the time to breakoff is comparable to the Rayleigh timescale $t_{R}=\sqrt{\rho R^{3} / \gamma}=8.153 \mu \mathrm{s}$ for the growth rate of the capillary instability. However, the increasing drop speed does lead to a slight increase in breakoff, which may be considered counterintuitive, but arises from the stabilizing effect of the extensional flow. 


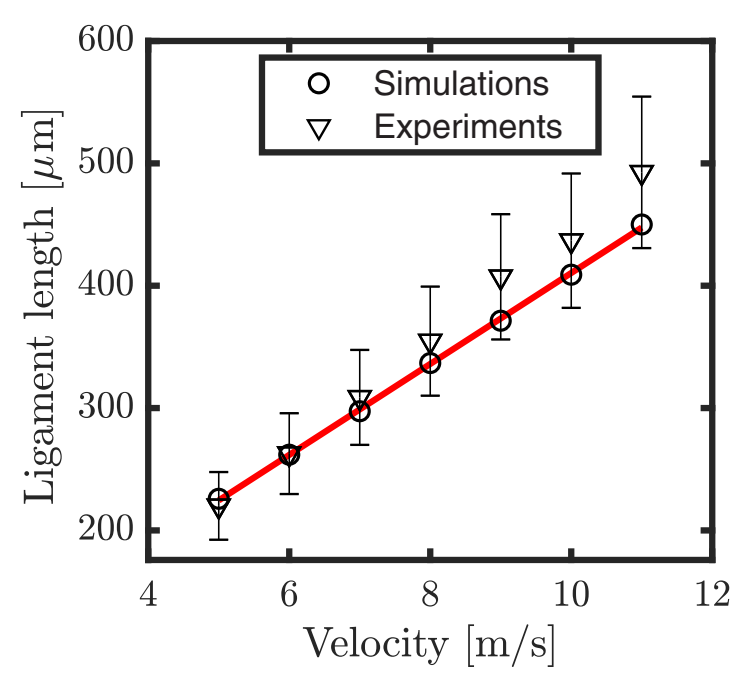

FIG. 7. Comparison of the ligament length at the point of breakoff as a function of drop velocity between simulations and experiments. The solid line is the linear fit to the simulation data. The ligament length increases linearly with the drop velocity.

A consequence of the breakoff time being only weakly dependent on the drop velocity is that the ligament length at breakoff grows approximately linearly in proportion to the drop velocity and can be many jet diameters in length. This is shown in Fig. 7, where we compare the ligament lengths at different drop velocities between the simulations and experiments. Due to the resolution of video images, there is an uncertainty in the ligament lengths measured from the experiments due to the size of the pixels. To establish a length scale, we count the number of pixels across the diameter of the droplet, which is known, and assume an error of plus and minus one pixel in this measurement. This provides a relative error for the ligament length measurements. It can be seen that the simulations and experiments agree within this error range. These results also agree with the observations in previous experimental work of Dong et al. [18].

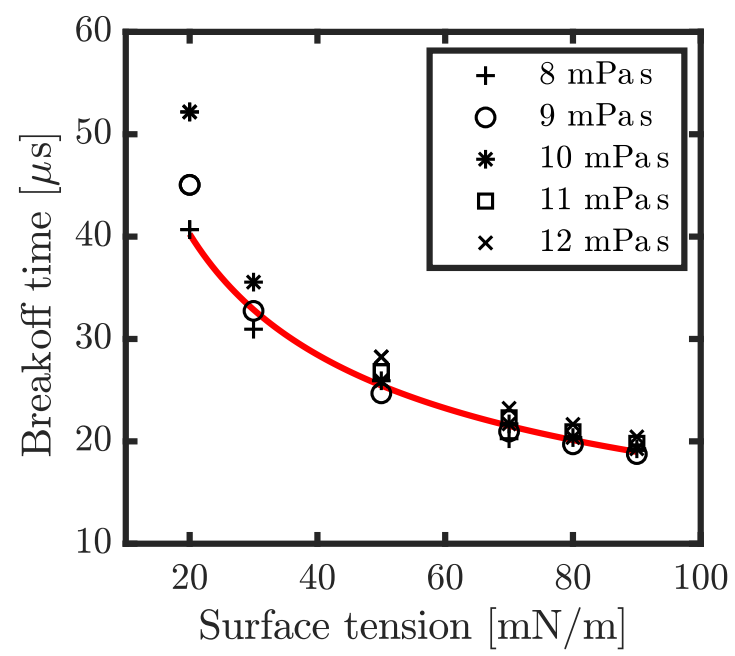

FIG. 8. Breakoff time for different fluids jetted at $7 \mathrm{~m} / \mathrm{s}$. The solid line shows the inverse square root of surface tension proportional to the Rayleigh timescale. 


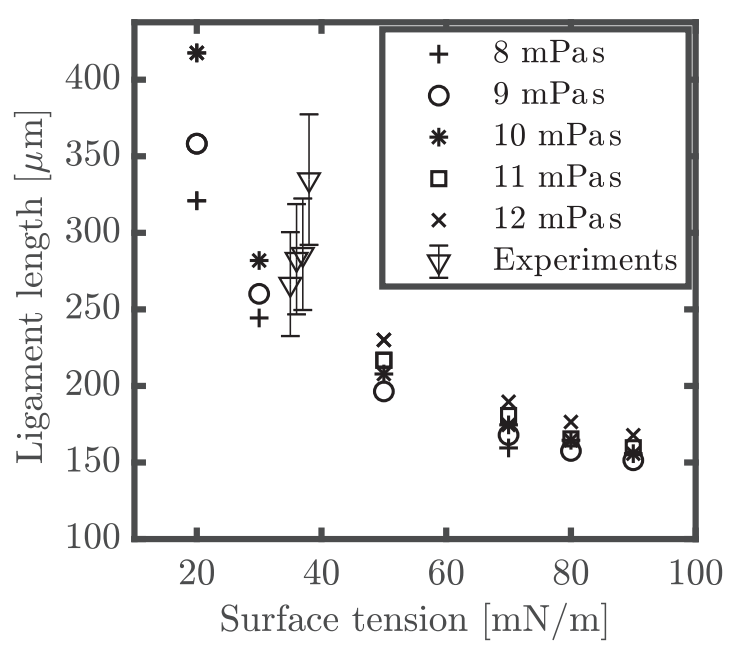

FIG. 9. Ligament length at breakoff time for different fluids jetted at $7 \mathrm{~m} / \mathrm{s}$. Measurements for the ligament length for different PPH-TPM blend are presented. The results agree well with the predicted simulation trend.

The results in Figs. 6 and 7 indicate that the time of breakoff is largely independent of drop velocity and that this time is comparable to the Rayleigh timescale. In contrast, the ligament length is directly proportional to droplet velocity over the typical operating range.

(a)
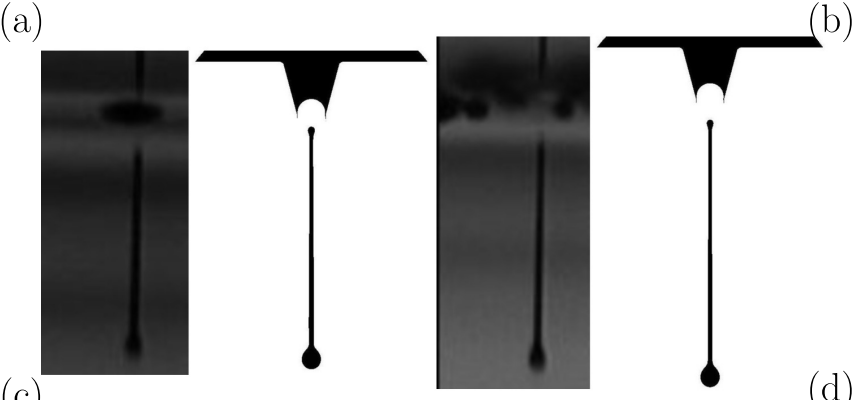

(c)
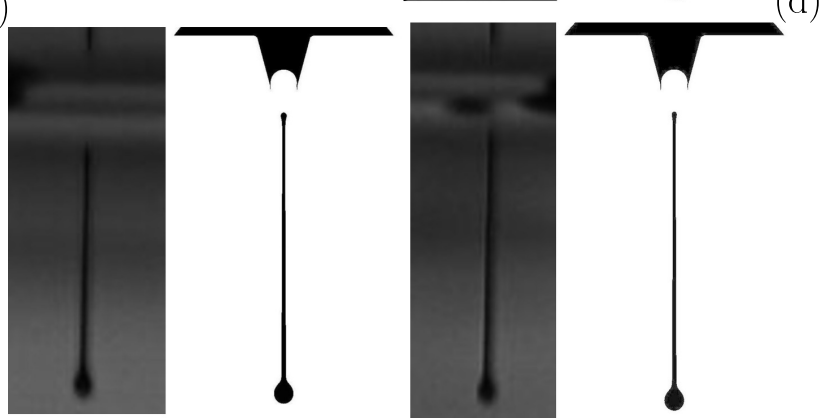

FIG. 10. Comparison between different PPH-TPM blends at $7 \mathrm{~m} / \mathrm{s}$. Shown on the left are experiments and on the right simulations: (a) $20 \%$ at $31 \mu \mathrm{s}$, (b) $40 \%$ at $35 \mu \mathrm{s}$, (c) $50 \%$ at $39 \mu \mathrm{s}$, and (d) $60 \%$ at $41 \mu \mathrm{s}$ with a ligament length of $22,25,30$, and $28 \mathrm{~mm}$, respectively. 
(a)

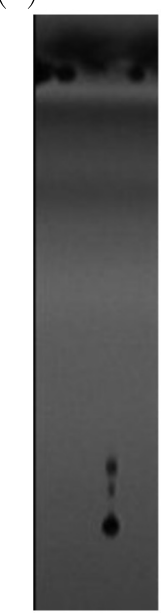

(c)

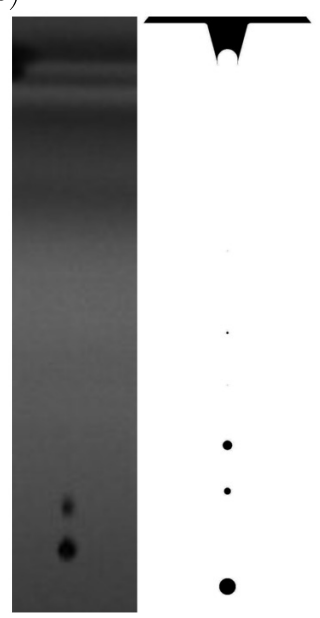

(b)
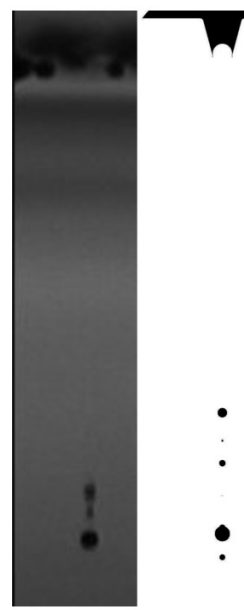

(d)
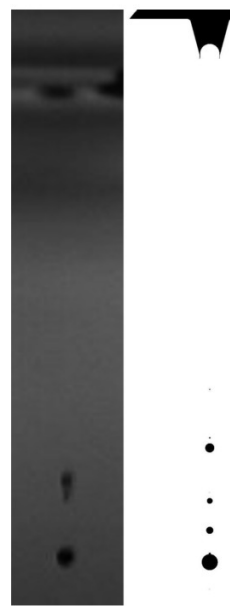

FIG. 11. Comparison between different PPH-TPM blends at $7 \mathrm{~m} / \mathrm{s}$. Shown on the left are experiments and on the right simulations: (a) $20 \%$ at $90 \mu \mathrm{s}$, (b) $40 \%$ at $94 \mu \mathrm{s}$, (c) $50 \%$ at $96 \mu \mathrm{s}$, and (d) $60 \%$ at $100 \mu \mathrm{s}$. Note the appearance of a satellite below the main drop at the longest elapsed time is an artefact of the simulation method and would in practice have coalesced with the main drop.

\section{Different fluid properties}

We now examine the effect of changing fluid properties when printing at a prescribed drop speed of $7 \mathrm{~m} / \mathrm{s}$. In these simulations the surface tension and viscosity were varied in the ranges of 20-90 mN/m and 8-12 mPa s, respectively. For each fluid the amplitude of the velocity waveform was adjusted until the desired drop velocity was achieved; however, the shape of the waveform was kept constant.

FIG. 12. Example of good jetting behavior. The two satellite droplets are moving faster than the main drop and so will merge with it. 


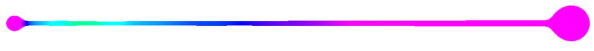

FIG. 13. Example of jet formation with a long stable ligament.

The results from Sec. III B suggest that while the drop speed is determined by the driving waveform, breakoff is primarily driven by surface-tension thinning. Figure 8 shows the first breakoff time for different ink properties. It can be seen that the first breakoff time is proportional to $\gamma^{-1 / 2}$, in agreement with the scaling suggested by the Rayleigh timescale. For this range of fluid viscosities there is also an increase in breakoff time with viscosity, particularly for low values of surface tension, which correspond to the highest values of Ohnesorge number. However, at low Ohnesorge number fluid viscosity is of secondary importance. Figure 9 shows the variation in ligament length, which mirrors the variations in breakoff time. Experimental measurements for the different PPH-TPM blends are also shown in Fig. 9, where we can see very good agreement with the trend predicted by the simulations.

Figures 10 and 11 compare the jetting behavior between experiments and simulations for different solutions of PPH and TPM with properties presented in Table I at constant drop speed $(7 \mathrm{~m} / \mathrm{s})$. The surface tensions of these mixtures are approximately the same $(36 \pm 2 \mathrm{mN} / \mathrm{m})$, while the viscosity ranges from 7.4 to $12.9 \mathrm{mPa}$. This increase in viscosity leads to a small increase in the breakoff time and the ligament length as shown in Fig. 10.

\section{Jetting behavior}

We now turn our attention to the subsequent drop formation after the drop and attached ligament have broken off from the nozzle in order to determine the range of fluid properties for which the jetted fluid forms into a single drop of the desired velocity before reaching the substrate. To do this, we ran a series of simulations with fluids of different viscosities and surface tensions through the same nozzle design at the same drop speed. For each of the simulations, the jetting behavior is characterized as being one of three types: (i) good, if a single drop is formed or a small number of fast satellites are formed that will coalesce with the main drop (Fig. 12); (ii) ligament, where the ligament becomes very long, typical of more viscous fluids with low surface tension (Fig. 13) (although these ligaments will eventually either retract into a single drop or break up into satellite drops, this can take longer than the time of flight of the drop before striking the substrate); and (iii) satellites, where the ligament breaks up into multiple smaller drops that do not coalesce with the main drop, typical of less viscous fluids (Fig. 14). In some cases it can be difficult to distinguish between the latter two as long ligaments will eventually break into satellites, so some cases are classified as ligament/satellite to indicate that a long ligament is formed that then breaks into satellites.

In Fig. 15 we show the range of fluid properties where these different behaviors are found in the form of a phase diagram in Ohnesorge-Reynolds space [8]. Since the Reynolds number is independent of the surface tension, changes in surface tension correspond to movements parallel to the vertical axis, whereas decreasing viscosity both reduces the Ohnesorge number and increases the Reynolds number.

The region of good behavior, sketched in the green cross region, is found to occur for Ohnesorge numbers in the range $0.2<\mathrm{Oh}<0.4$. We did not explore Ohnesorge numbers less than 0.2 as this was outside the range of realistic values of surface tension and viscosity for our chosen nozzle radius. This is consistent with the suggested jettability range of $0.1<\mathrm{Oh}<1$ [8,28]. However, we

FIG. 14. Example of drop formation where multiple satellite drops are formed that will not merge with the main drop. 


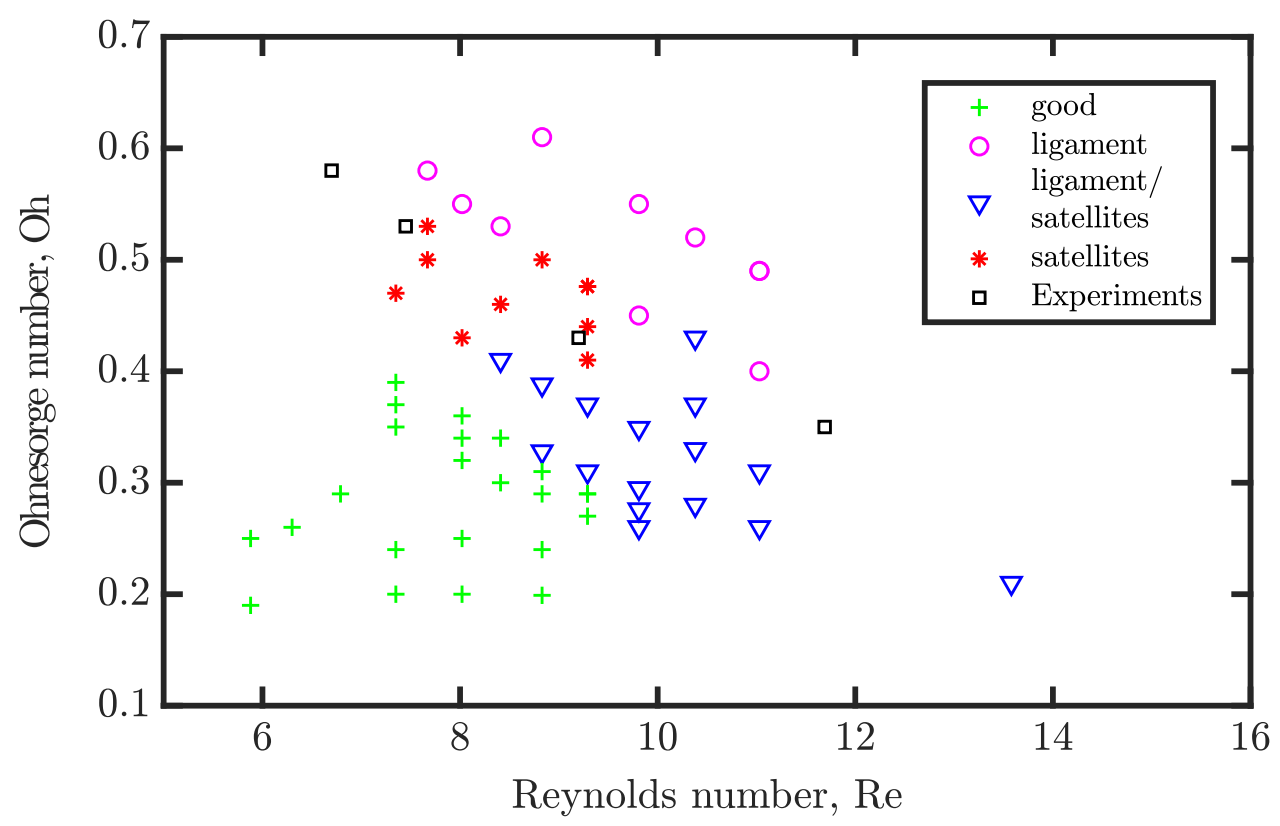

FIG. 15. Jetting behavior diagram showing the location of different classes of drop formation in terms of Oh and Re.

find an additional restriction to $\operatorname{Re}<10$. At higher Reynolds numbers, we always observe satellite formation even at $\mathrm{Oh}=0.3$. This restriction is distinct from the Reynolds number limit discussed in the literature [1,8], which arises from splashing on impact with the substrate. In this case the restriction arises from the length (and hence aspect ratio) of the ligament formed behind the drop. At a fixed value of $\mathrm{Oh}$ an increase in Reynolds number corresponds to an increase in drop velocity and hence ligament length, as shown in Fig. 7. Even at moderate values of Oh, ligaments of very high aspect ratio will break up to form satellite drops [7,16]. At values of $\mathrm{Oh}>0.4$, fluid viscosity significantly retards the breakoff of the drop from the nozzle, as seen in Fig. 8, for low surface tension and high viscosity, resulting in the formation of a long ligament.

\section{CONCLUSION}

We have presented results from numerical simulations and experiments of droplet formation in an industrial DOD printhead. We found good agreement in the evolution of droplet shapes between the experiments and simulations, despite the uncertainty in determining the precise shape of the drive waveform.

This level of agreement allows us to use the simulations to analyze the mechanisms driving droplet formation. While the drive is responsible for the momentum of the drop, it is surface tension that it responsible for the breakoff from the nozzle and is found to be proportional to the Rayleigh timescale for the growth of capillary instabilities. Indeed, the effect of increasing drop velocity is to increase slightly the breakoff time. As a consequence, the length of the ligament behind the drop grows in proportion to the drop speed. The length of this ligament limits the range of drop velocities for which the ligament will retract into the main drop before it breaks up to form satellite drops. For the particular printhead and drive in our study we found that this limits the range of fluids that produce satellite-free drops to Reynolds numbers below 10, irrespective of the Ohnesorge number of the fluid. This provides an additional limit of the window of "printable" fluids in addition to the restriction on the values of the Ohnesorge number. 
Our results also confirm that an Ohnesorge number of around 0.3 is optimal for controlling satellites in DOD printing. Although a larger Ohnesorge number would further stabilize the retracting ligament, the increased viscosity also acts to delay the breakoff from the nozzle and so increases the length of the ligament.

\section{ACKNOWLEDGMENTS}

This work was supported by the Engineering and Physical Sciences Research Council Centre for Doctoral Training in Fluid Dynamics at the University of Leeds under Grant No. EP/L01615X/1. The authors would like to thank Ricoh for supporting the project.

[1] S. D. Hoath, Fundamentals of Inkjet Printing: The Science of Inkjet and Droplets (Wiley, New York, 2016).

[2] G. D. Martin, S. D. Hoath, and I. M. Hutchings, Inkjet printing-the physics of manipulating liquid jets and drops, J. Phys.: Conf. Ser. 105, 012001 (2008).

[3] I. Hutchings, G. Martin, and S. Hoath, High speed imaging and analysis of jet and drop formation, J. Imaging Sci. Technol. 51, 438 (2007).

[4] C. McIlroy, Complex inkjets: Particles, polymers and non-linear driving, Ph.D. thesis, University of Leeds, 2014.

[5] J. Plateau, Experimental and Theoretical Statics of Liquids Subject to Molecular Forces Only (GauthierVillars, Paris, 1873).

[6] L. Rayleigh, On the instability of jets, Proc. London Math. Soc. 1, 4 (1878).

[7] S. Hoath, J. Jung, and I. M. Hutchings, A simple criterion for filament break-up in drop-on-demand inkjet printing, Phys. Fluids 25, 021701 (2013).

[8] G. H. McKinley and M. Renardy, Wolfgang von Ohnesorge, Phys. Fluids 23, 127101 (2011).

[9] B. Derby, Inkjet printing of functional and structural materials: Fluid property requirements, feature stability, and resolution, Annu. Rev. Mater. Res. 40, 395 (2010).

[10] O. A. Basaran, H. Gao, and P. P. Bhat, Nonstandard inkjets, Annu. Rev. Fluid Mech. 45, 85 (2013).

[11] N. F. Morrison and O. G. Harlen, Viscoelasticity in inkjet printing, Rheol. Acta 49, 619 (2010).

[12] S. D. Hoath, O. G. Harlen, and I. M. Hutchings, Jetting behavior of polymer solutions in drop-on-demand inkjet printing, J. Rheol. 56, 1109 (2012).

[13] J. de Jong, G. de Bruin, H. Reinten, M. van den Berg, H. Wijshoff, M. Versluis, and D. Lohse, Air entrapment in piezo-driven inkjet printheads, J. Acoust. Soc. Am. 120, 1257 (2006).

[14] R. Schulkes, The contraction of liquid filaments, J. Fluid Mech. 309, 277 (1996).

[15] P. K. Notz and O. A. Basaran, Dynamics and breakup of a contracting liquid filament, J. Fluid Mech. 512, 223 (2004).

[16] A. A. Castrejón-Pita, J. R. Castrejón-Pita, and I. M. Hutchings, Breakup of Liquid Filaments, Phys. Rev. Lett. 108, 074506 (2012).

[17] C. R. Anthony, P. M. Kamat, M. T. Harris, and O. A. Basaran, Dynamics of contracting filaments, Phys. Rev. Fluids 4, 093601 (2019).

[18] H. Dong, W. W. Carr, and J. F. Morris, An experimental study of drop-on-demand drop formation, Phys. Fluids 18, 072102 (2006).

[19] G. D. Martin, I. M. Hutchings, and S. D. Hoath, in Proceedings of the Digital Fabrication Conference, Denver, 2006 (Society for Imaging Science and Technology, Springfield, 2006), pp. 95-98.

[20] R. Li, N. Ashgriz, S. Chandra, and J. R. Andrews, Contraction of free liquid ligaments, AIChE J. 54, 3084 (2008).

[21] M. Tjahjadi, H. A. Stone, and J. M. Ottino, Satellite and subsatellite formation in capillary breakup, J. Fluid Mech. 243, 297 (1992).

[22] F. J. Muzzio, M. Tjahjadi, and J. M. Ottino, Self-Similar Drop-Size Distributions Produced by Breakup in Chaotic Flows, Phys. Rev. Lett. 67, 54 (1991). 
[23] J. R. Castrejón-Pita, N. F. Morrison, O. G. Harlen, G. D. Martin, and I. M. Hutchings, Experiments and Lagrangian simulations on the formation of droplets in drop-on-demand mode, Phys. Rev. E 83, 036306 (2011).

[24] S. Wang, Y. Zhong, and H. Fang, Deformation characteristics of a single droplet driven by a piezoelectric nozzle of the drop-on-demand inkjet system, J. Fluid Mech. 869, 634 (2019).

[25] O. Harlen, J. Rallison, and P. Szabo, A split Lagrangian-Eulerian method for simulating transient viscoelastic flows, J. Non-Newtonian Fluid Mech. 60, 81 (1995).

[26] H. Westborg and O. Hassager, Creeping motion of long bubbles and drops in capillary tubes, J. Colloid Interface Sci. 133, 135 (1989).

[27] J. Eggers, Universal Pinching of 3D Axisymmetric Free-Surface Flow, Phys. Rev. Lett. 71, 3458 (1993).

[28] I. M. Hutchings and G. D. Martin, Inkjet Technology for Digital Fabrication (Wiley, New York, 2012).

[29] A. U. Chen and O. A. Basaran, A new method for significantly reducing drop radius without reducing nozzle radius in drop-on-demand drop production, Phys. Fluids 14, L1 (2002).

[30] H. Dong, W. W. Carr, and J. F. Morris, Visualization of drop-on-demand inkjet: Drop formation and deposition, Rev. Sci. Instrum. 77, 085101 (2006). 